\title{
Comparing Quality of Intimate Relationship and Anger Experience in Patients with Borderline Personality
} and Bipolar-II Disorders

Zohreh Khosravi, Parvin Rahmatinejad", Azam Farah Bijari

Department of Psychology, Alzahra University, Tehran, Iran

\section{A BSTRACT}

Introduction: Because of the high similarity of symptoms and clinical characteristics between borderline personality and bipolar spectrum disorders particularly bipolar-II disorder, some investigators reclassified borderline personality disorder on bipolar spectrum and considered common etiology for two disorders. In the present study in order to evaluate this hypothesis, intimate relationship and anger experience in borderline personality and bipolar-II disorders were compared. Materials and Methods: A sample consists of 27 borderline personality and 31 bipolar-II disorder outpatients, who were referred to the Psychiatric Ward of Kamkar-arabnia hospital in Qom, Iran were selected through convenience sampling method. They answered to "Quality of relationship inventory" (QRI) and "Multidimensional Anger Inventory" (MAI). The mean of variables in both groups were compared by Independent T-test. Results: The results of Independent T-test showed that there was no significant difference between two groups in quality of intimate relationship and anger experience. However, borderline and bipolar-II patients had a significant difference in conflict subscale of QRI and anger arousal subscale of MAI. Compared to the bipolar-II patients, borderline patients were obtained higher scores on both subscales. Conclusion: No significant difference between quality of intimate relationship and anger experience in borderline and bipolar-II groups may suggest authenticity of the hypothesis that borderline disorder belongs to bipolar spectrum and both disorders might have common etiology.

*Corresponding Author: Parvin Rahmatinejad

E-mail: Rahmatinejadp@yahoo.com 


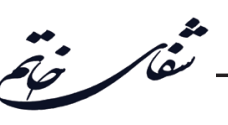

مقايسُٔ كيفيت رابطُٔ صميمانه و تجربئ خشم در بيماران مبتلا به اختلالات شخصيت مرزى و

\section{دوقطبى نوع II}

زهره خسروى، يروين رحمتىنزاد"، اعظم فرح بيجارى

كروه روانشناسى، دانشعاه الزهرا، تهران، ايران

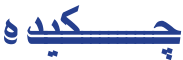

كليد وازهها:

مقدمه: به دليل شباهت بالاى نشانهها و مشخصات بالينى بين اختلالات طيف شخصيت مرزى و دوقطبى اختى

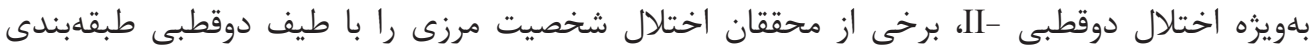

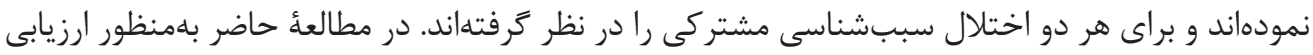

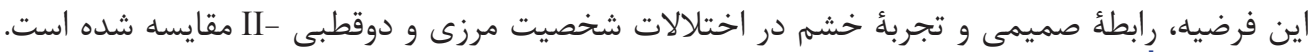

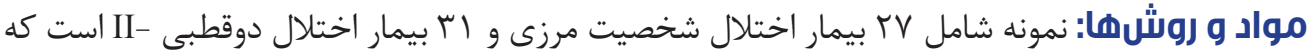

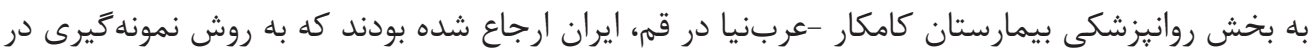

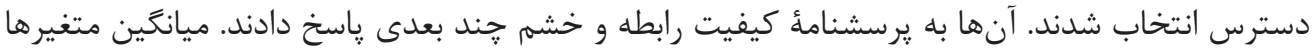

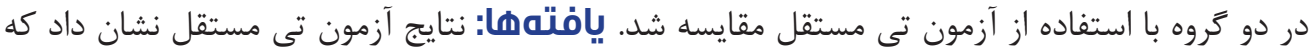

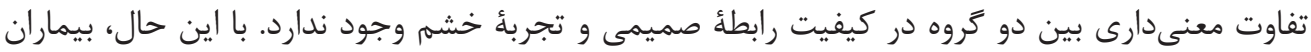

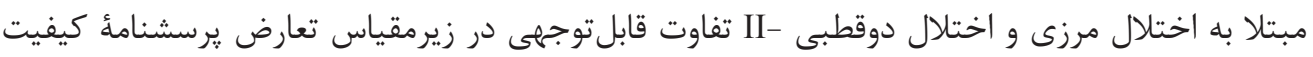

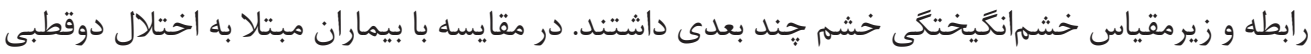

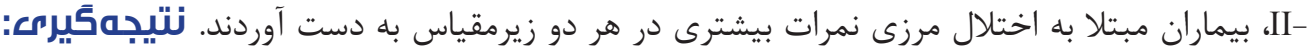

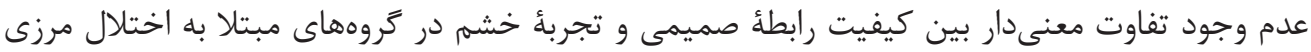

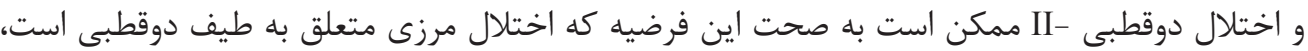

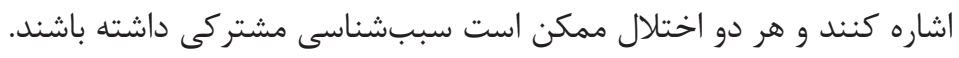

" نويسنده مسئول: يروين رحمتىنزاد آدرس الكترونيكى: Rahmatinejadp@yahoo.com 
در راستاى اين فرضيه مىتوان به توصيفات بالينى و تجربى

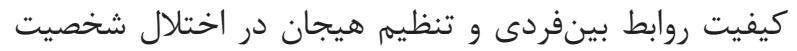

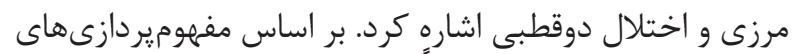

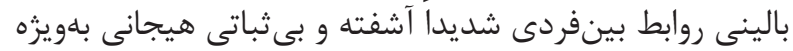

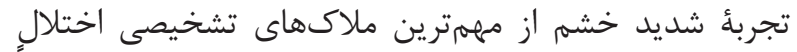

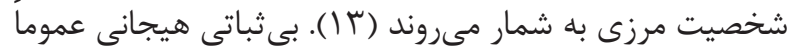

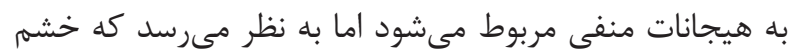

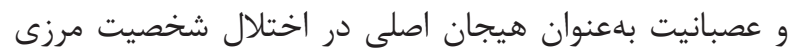

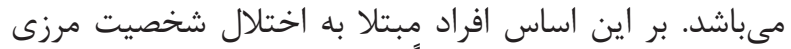

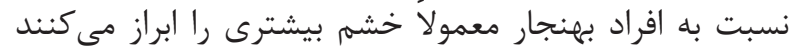
(F)

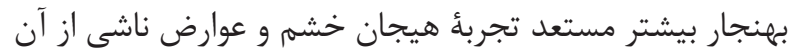

هستند (ه (1).

تحقيقاتى كه در اين زمينه صورت كرفته است اهميت هيجان

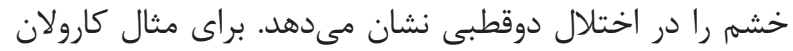

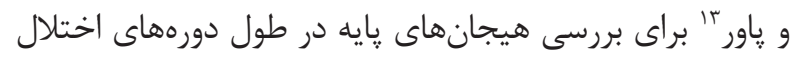

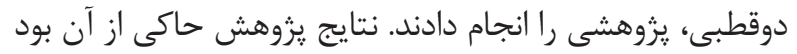

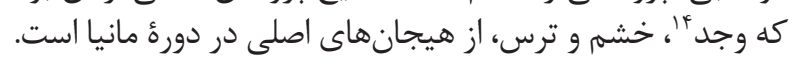

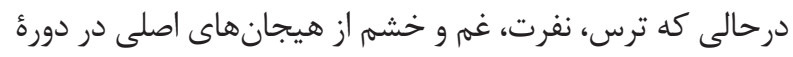

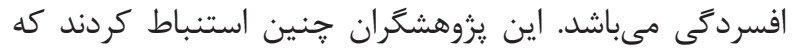

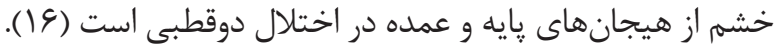

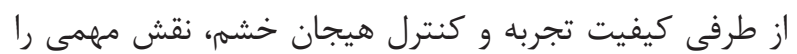

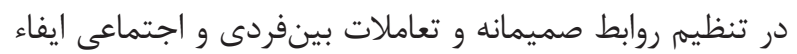

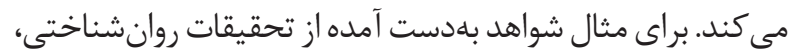

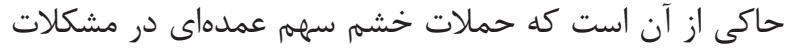

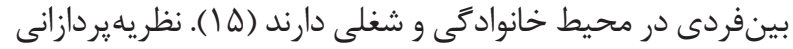

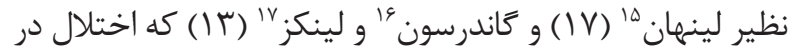

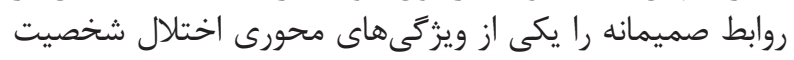

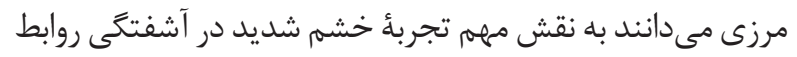

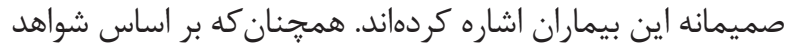

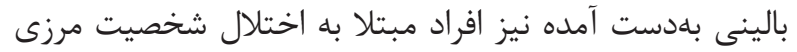

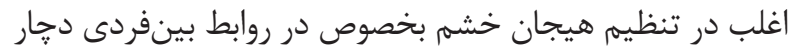

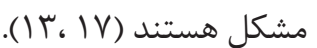

بسيارى از افراد مبتلا به اختلال دوقطبى نيز مشكلاتى را راند

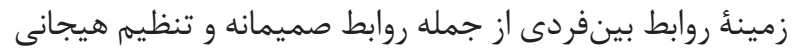

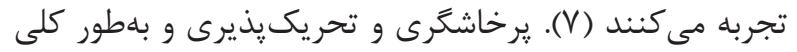

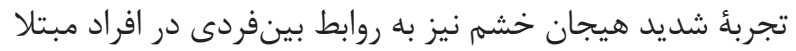

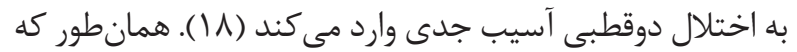

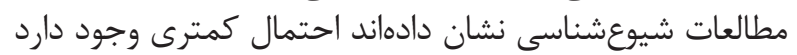

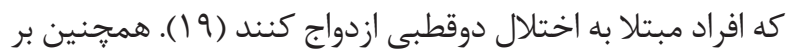

${ }^{1}$ Borderline personality disorder

${ }^{2}$ Affective disorder

${ }^{3}$ Bipolar disorder-II

${ }^{4}$ Mania

${ }^{5}$ Mixed states

${ }^{6}$ Comorbidity

${ }^{7}$ Akiskal

${ }^{8}$ Bipolar disorder spectrum

${ }^{9}$ Atypical depression

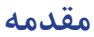

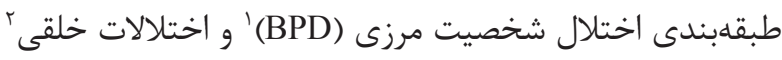

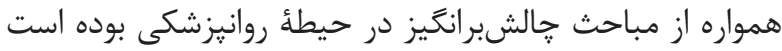

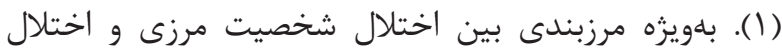

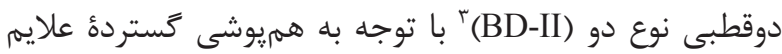

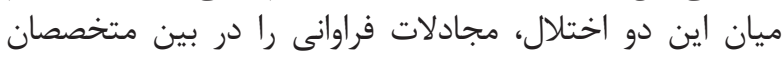

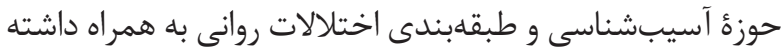

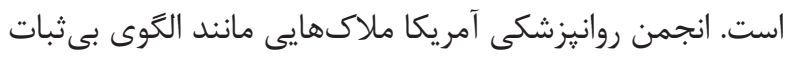

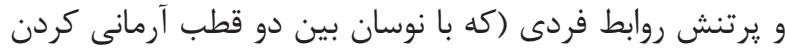

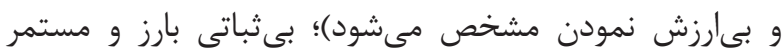

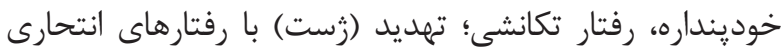

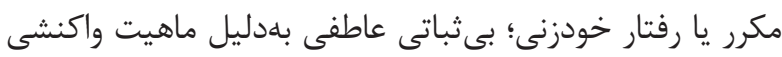

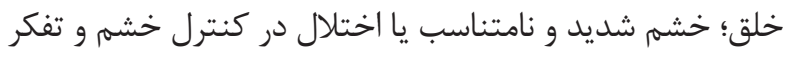

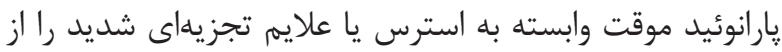

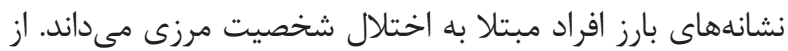

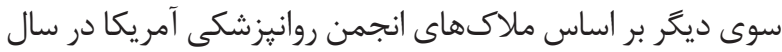

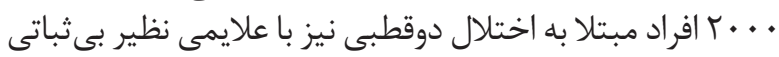

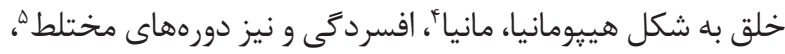

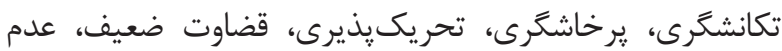

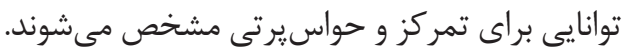

با توجه به شباهت زياد علايم دو اختلال، مطرح شدن مباحث

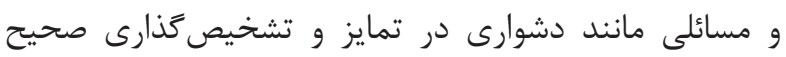

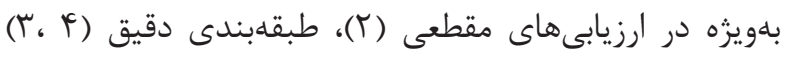

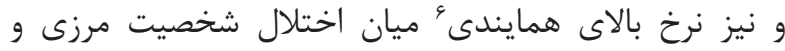

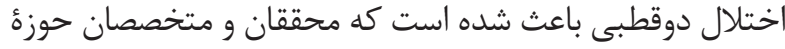

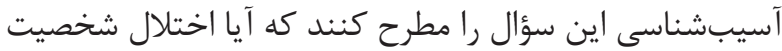

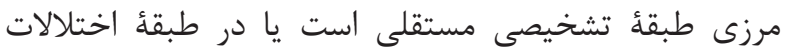
خلقى مفهوميردازى دقيقترى خواهد داشت است (^-ه).

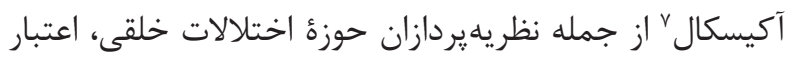

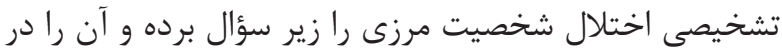

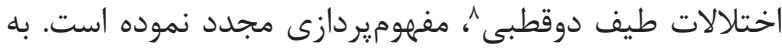

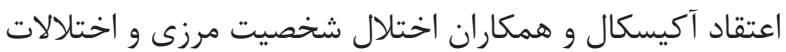

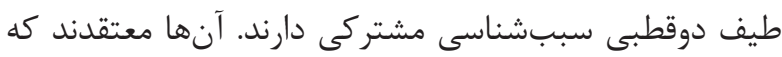

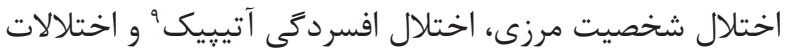
طيف دوقطبى - II- در بستر زيستى -روانى مشتركى كه همان آنسان

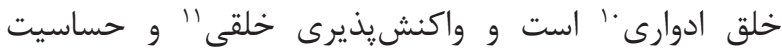

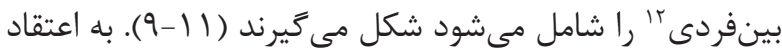

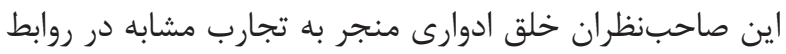

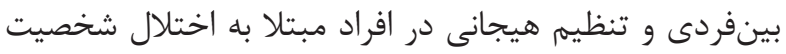

مرزى و اختلالات طيف دوقطبى مى شود (r) (I).

\footnotetext{
${ }^{10}$ Cyclothymic temperament

${ }^{11}$ Affective reactivity

${ }^{12}$ Interpersonal sensitivity

${ }^{13}$ Carolan and Power

${ }^{14}$ Happiness

${ }^{15}$ Linehan

${ }^{16}$ Gunderson

${ }^{17}$ Links
} 


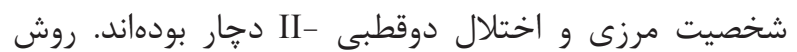

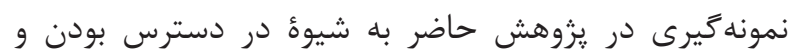

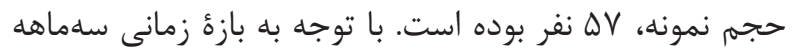

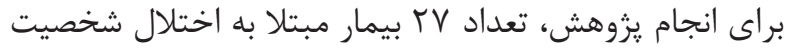

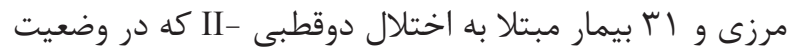

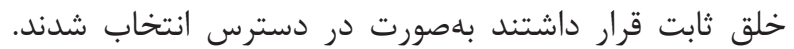

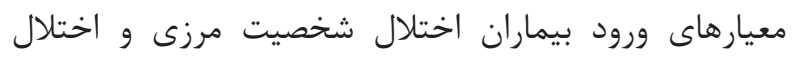

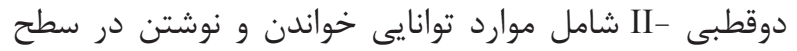

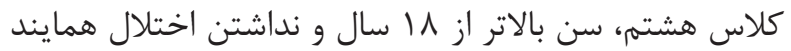

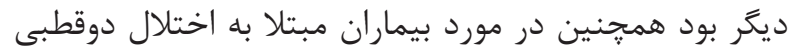

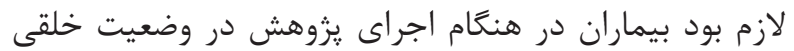

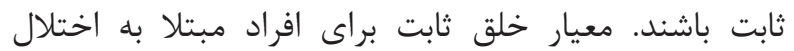

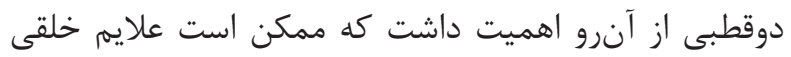

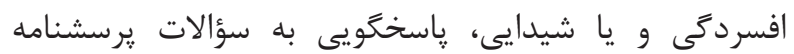

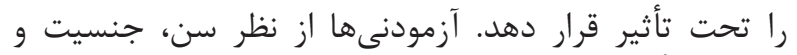

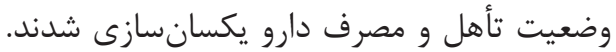

روند اجراى هُخوهش

يس از كسب مجوز از دانشعاه علوم :زئشى قم اقدام به

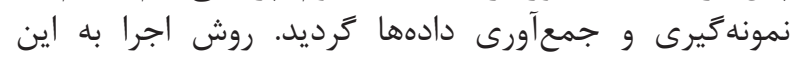

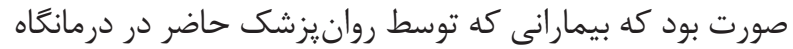

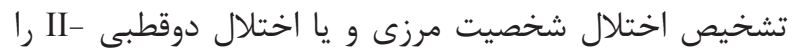

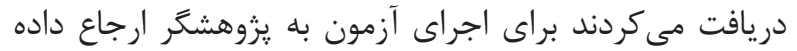

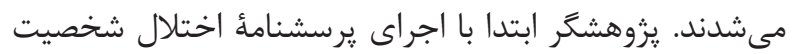

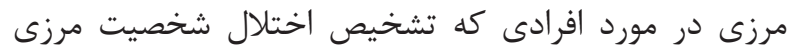

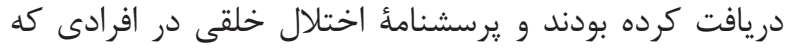

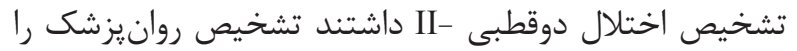

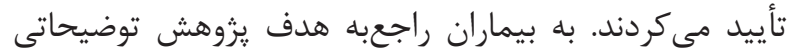

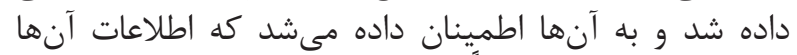

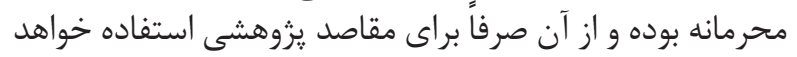

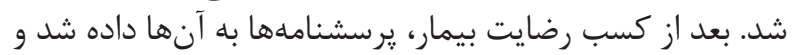

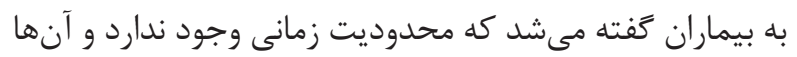

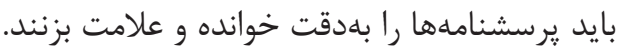

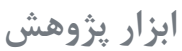

1- - يرسشنامة شخصيت مرزى

ليشنر ينغ 19 اين يرسشنامه را بامنظور سنجش اختلال شخصيت مرزى بر اساس ملاكهاى DSM-IV-TR و ور صفات شخصيت

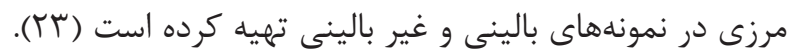

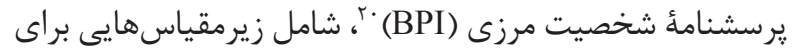

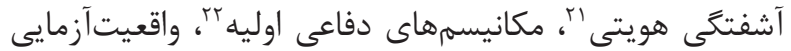

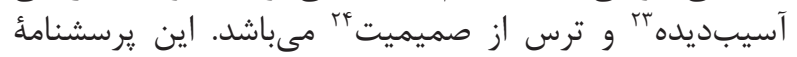

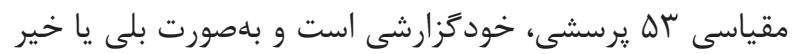

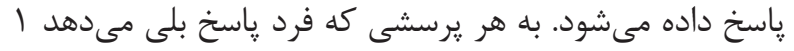
نمره داده مىشود.

\footnotetext{
${ }^{18}$ Marital

${ }^{19}$ Leichsenring

${ }^{20}$ Borderline personality inventory

${ }^{21}$ Identity diffusion
}

اساس اين يافتهها بيماران مبتلا به اختلال دوقطبى، اغلب روابط

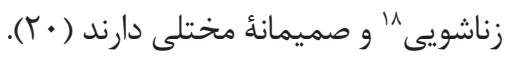

مرور مطالعات ييشين حاكى از آن است كه در تحقيقات

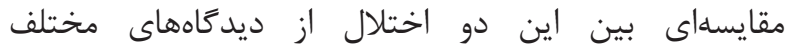

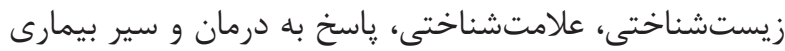

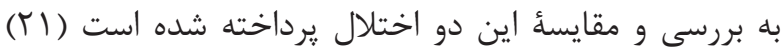

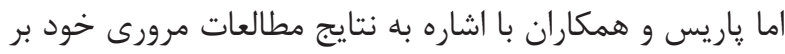
طرحريزى يزوهش هاى مختلف براى مقايسه و بررسى اخدان اختلال

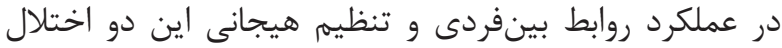
تأكيد كردهاند.

به اعتقاد اين يزوهشگران تحقيقات بيشتر در اين زمينه، از آن آن إنان

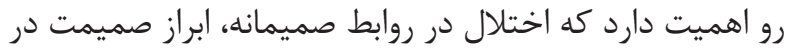

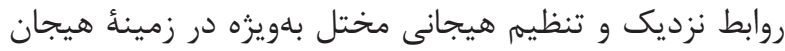

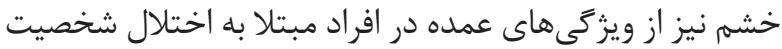

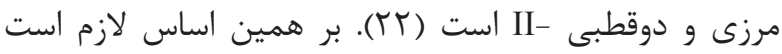

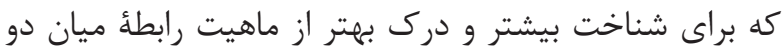

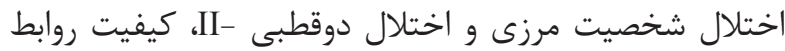

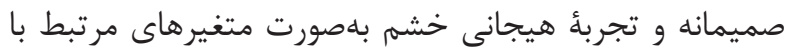

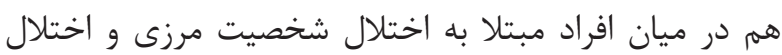
دوقطبى -III، مورد بررسى و مقايسه قرار گيرد.

باطور كلى اين يزوهش، به بررسى و مقايسٔ كيفيت روابط

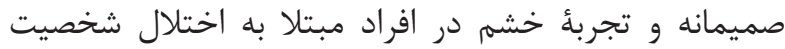

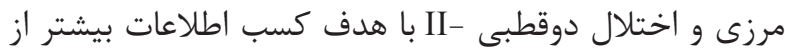

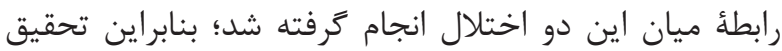

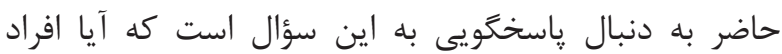

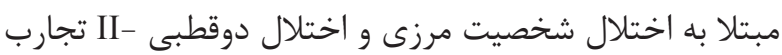

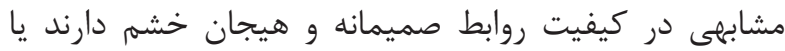

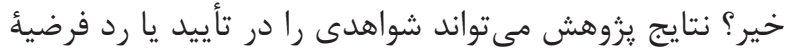

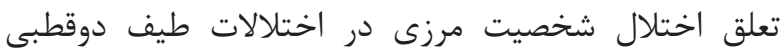

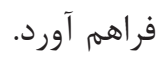

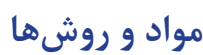

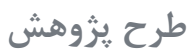

يثوهش حاضر بر اساس هدف، از نوع بنيادى و بر اساس روش إش

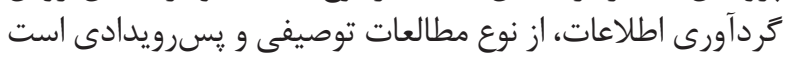

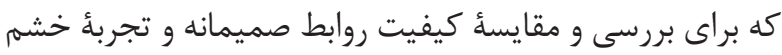
II- در افراد مبتلا به اختلال شخصيت مرزى ور اختئل صورت پيذيرفته است.

$$
\text { آزمودنى ها }
$$

جامعةُ آمارى يزوهش را كليئ بيماران مراجعه كننده به درمانگاه

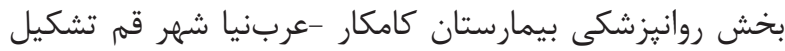

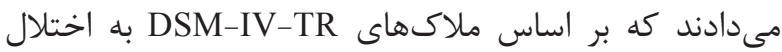

\footnotetext{
${ }^{22}$ Primitive defense mechanisms

${ }^{23}$ Impaired reality testing

${ }^{24}$ Fear of closeness
} 
و تنظيم مجدد و تغييراتى در شكل و محتواى فرم ترجمه شده

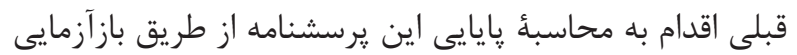

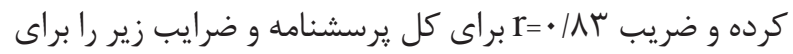

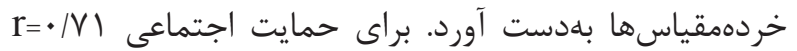

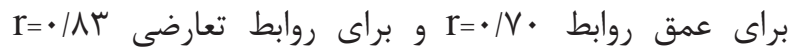
محاسبه شد.

F - يرسشنامهُ خشم جندبعدى

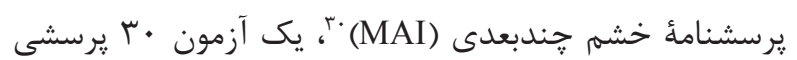

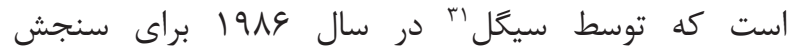

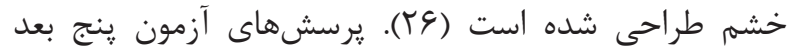

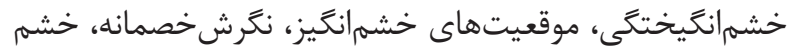

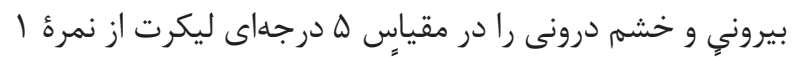

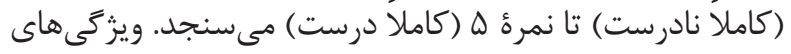

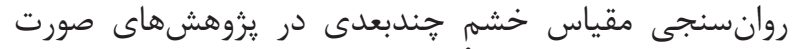

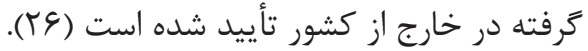

در فرم فارسى اين مقياس، آلفاى كرونباخ يرسشهاى هر يك از

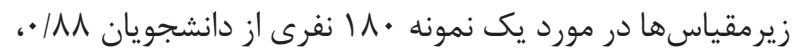

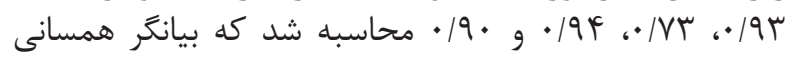

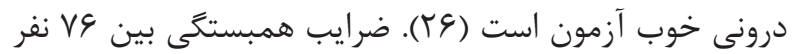

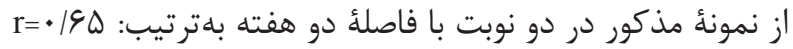

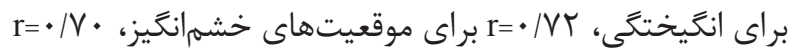

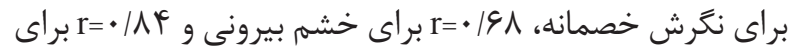

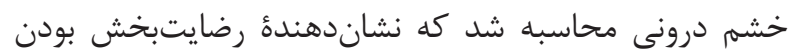
يايايى بازآزمون اين مقياس است.

تحليل دادهها با استفاده از نرمافزار 16 SPSS، در دو سطح

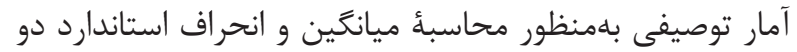

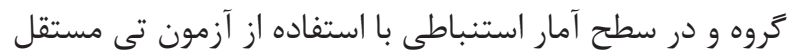
صورت گ ترفت.

يافته ها

اطلاعات بلهدست آمده از خصوصيات جمعيتشناختى دو گرووه

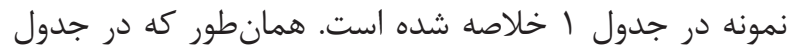

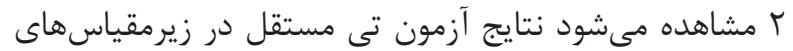

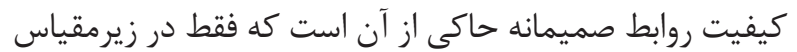

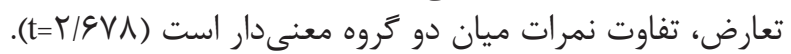

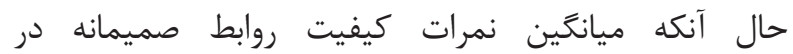

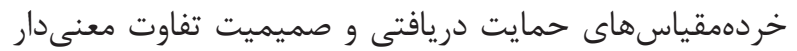

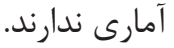

با توجه به جدول ب بر اساس نتايج آزمون تى مستقل در

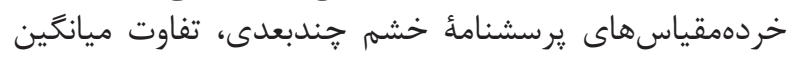

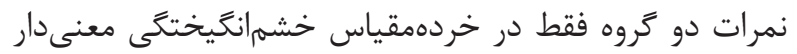

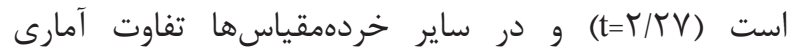
معنى دارى مشاهده نشد.

${ }^{25}$ Sensitivity

${ }^{26}$ Mood disorder questionnaire

${ }^{27}$ Hirschfeld

${ }^{28}$ Quality of relationship inventory
نتايج زئوهش ليشنرينگ بررسى همسانى درونى و اعتبار

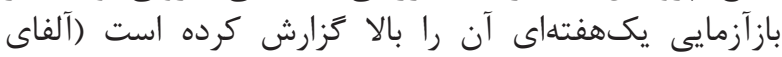

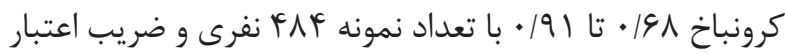

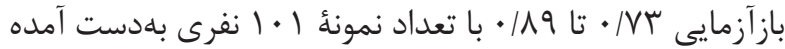

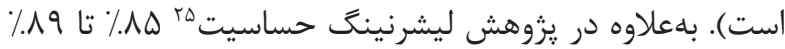
و ويزز

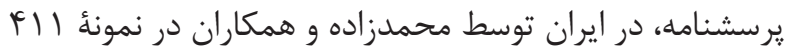

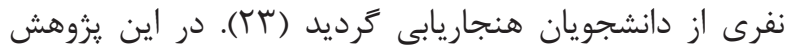

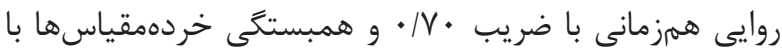

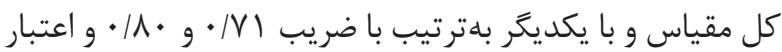

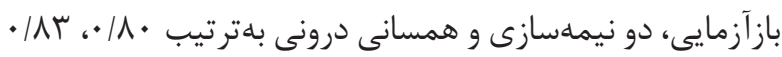

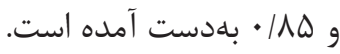

\section{r- برسشنامهُ اختلال خلقى}

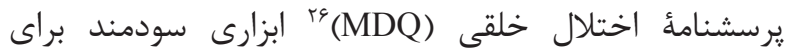

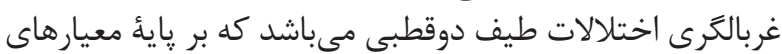

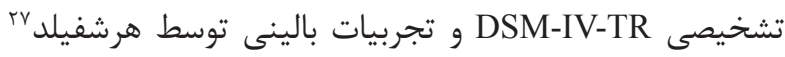

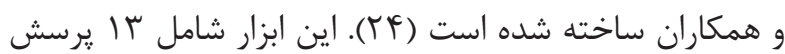

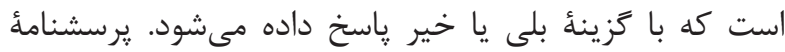

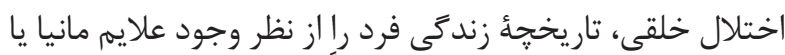

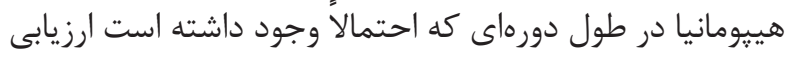

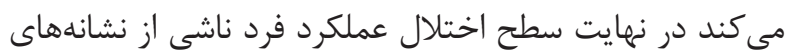

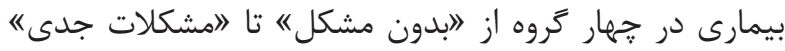

رتبـابندى مىشود.

در بررسىهاى انجام شده توسط هرشفيلد و همكاران براى

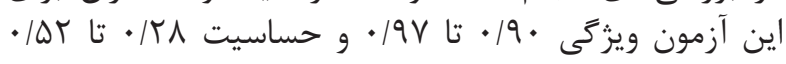

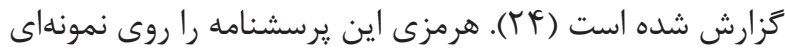

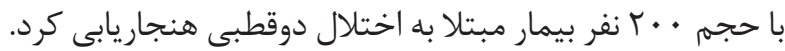
״ايايى يرسشنامه با استفاده از آزمون -باز آزمون

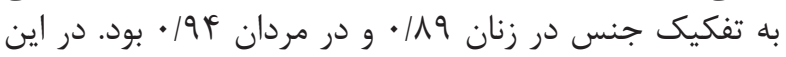

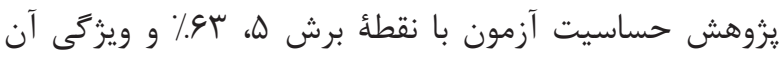

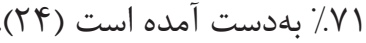

r- - برسشنامةٔ كيفيت روابط

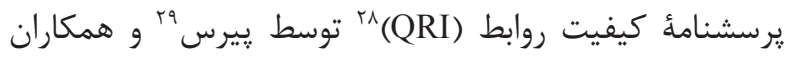

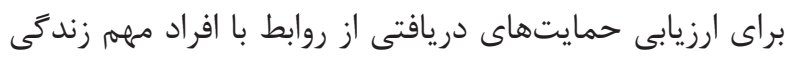

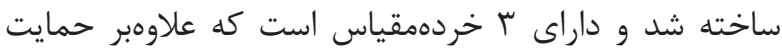

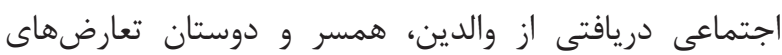

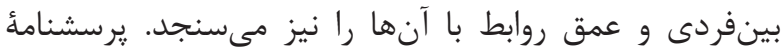

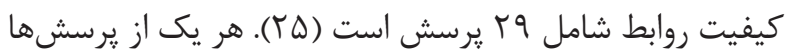

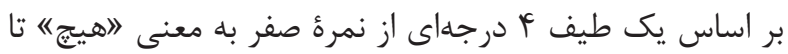

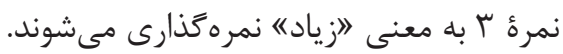

اعتبار و پايايى گرسشنامه كيفيت روابط در تحقيقات خارجى

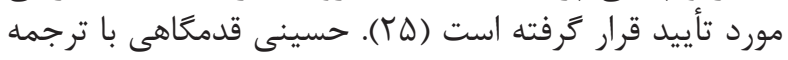

${ }^{29}$ Pierce

${ }^{30}$ Multidimensional anger inventory

${ }^{31}$ Siegel 


\section{BD-II}

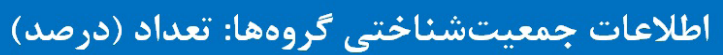

\begin{tabular}{|c|c|c|c|}
\hline$\Pi(\% / \Delta r / T)$ & $T V(\%$ FN/A) & & فراوانى \\
\hline $18(\% \& \wedge / f)$ & $1 \Delta(\% / \Delta \Delta / \varepsilon)$ & زن & حنس \\
\hline $1 \Delta(\% . \Delta 1 / \varepsilon)$ & $I F(\% / F F / F)$ & 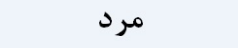 & \\
\hline $9(\% / 19 / 4)$ & $F(\% / . \mid \Lambda)$ & كمتر از •r سال & \\
\hline $19(91 / 7)$ & $1 \Delta(\% / \Delta \Delta / \Delta)$ & بين •r تا •F سال & سن \\
\hline$q(\% / 19 / T)$ & $\wedge(\%$ ห / /q) & بالاى ·F سال & \\
\hline $19(\% .91 / r)$ & $19(\% / v \cdot / F)$ & مجرد & \\
\hline $1 \cdot(\% / r / T)$ & $r(\% / 11 / 1)$ & متأهل & وضعيت تأه \\
\hline 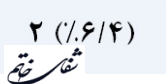 & $\Delta(\% / 1 \Lambda / \Delta)$ & مطلقه & \\
\hline
\end{tabular}

جدول r- نتايج آزمون تى مستقل در خردمقياسهاى كيفيت روابط بين دو گروه نموند.

\begin{tabular}{|c|c|c|c|c|c|}
\hline$P$ & $\mathbf{t}$ & انحراف استاندارد & ميانگين & Fر Fهو & متغيرها \\
\hline.$|9|$ & $-\cdot / \Delta T$ & $\begin{array}{l}\text { V/rT } \\
\text { g/gVA }\end{array}$ & $\begin{array}{l}11 / \Delta . \\
\| r / r \wedge\end{array}$ & $\begin{array}{c}\text { BPD } \\
\text { BD-II }\end{array}$ & ن تىى \\
\hline .1 .1 & r/9VA & $\begin{array}{l}\Delta / \Lambda) \\
V / \Lambda \wedge\end{array}$ & $\begin{array}{l}r \Delta / F \Lambda \\
r \cdot / \Delta F\end{array}$ & $\begin{array}{c}\text { BPD } \\
\text { BD-II }\end{array}$ & تعارض \\
\hline ثم ثة & $1 / \cdot v$ & $\begin{array}{l}r / . G \\
\Delta / V T\end{array}$ & $\begin{array}{l}\mid \Delta / V \Delta \\
\mid r / \Delta r\end{array}$ & $\begin{array}{c}\text { BPD } \\
\text { BD-II }\end{array}$ & صميميت \\
\hline
\end{tabular}

جدول بـ- نتايج آزمون تى مستقل در خردهمقياسهاى خشم خندبعدى بين دو كروه نمونه.

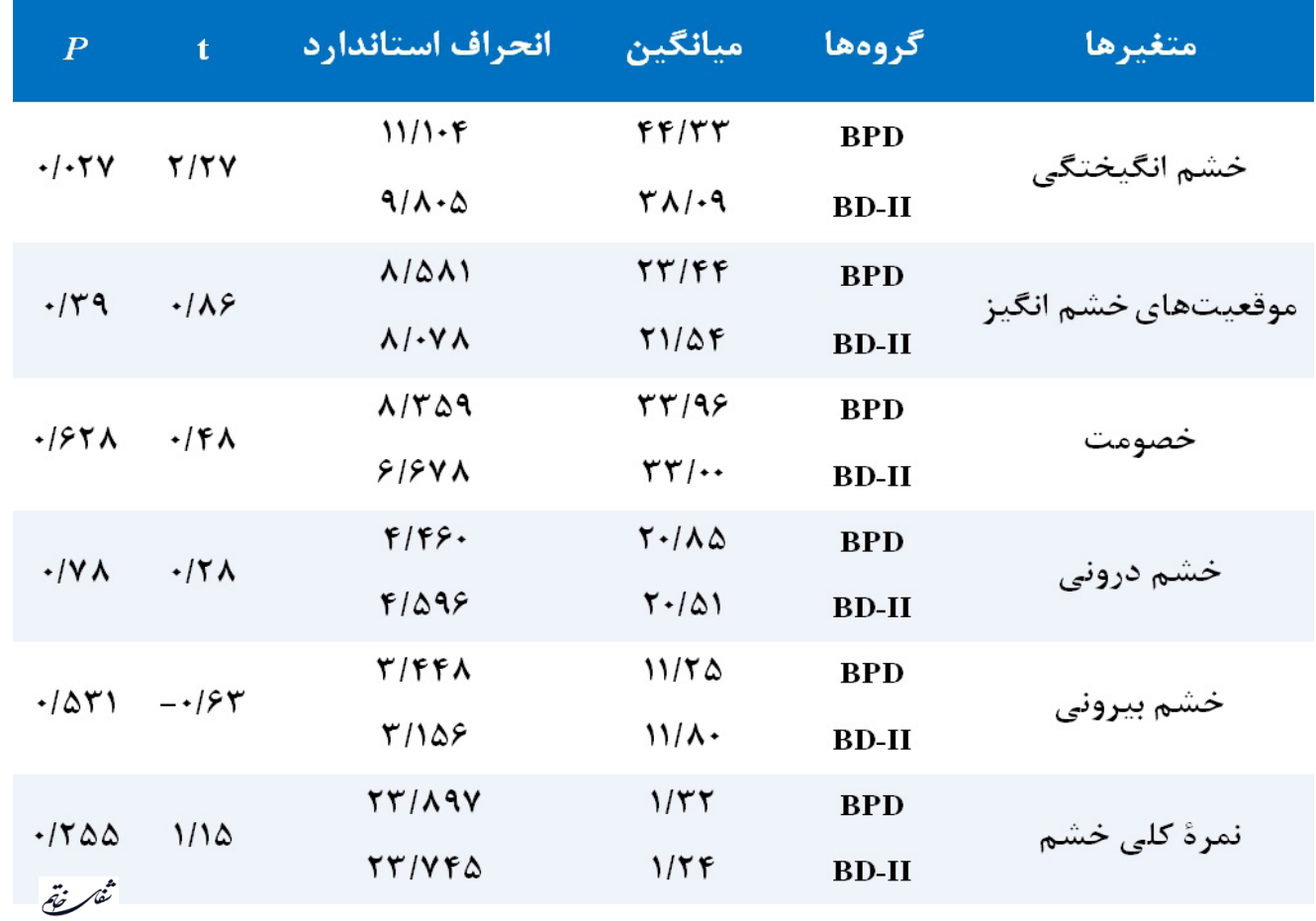


كه شدت خشم را بين دو گروه مقايسه كردهاند نيز نشان داد

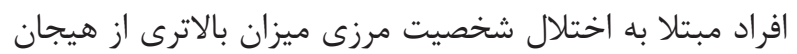

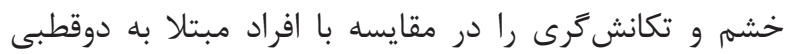

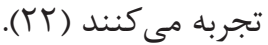

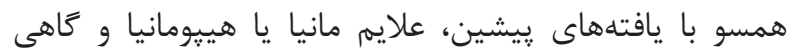

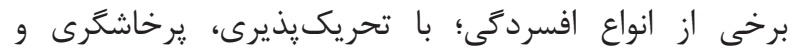

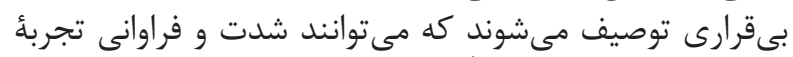

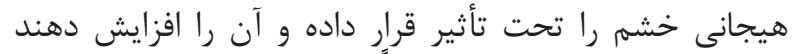

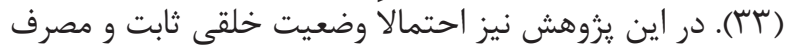

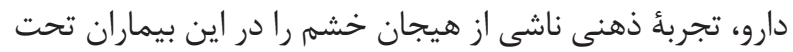

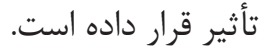

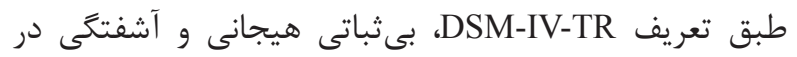

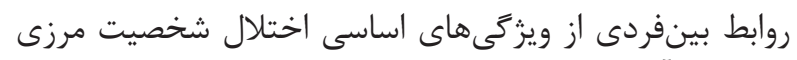

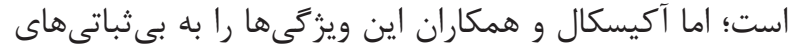

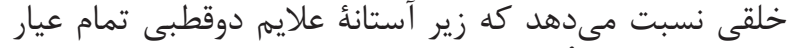

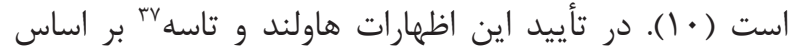

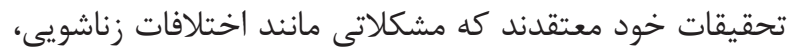

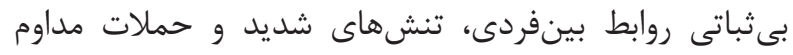

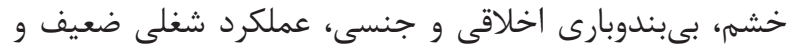

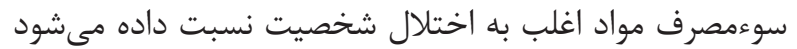

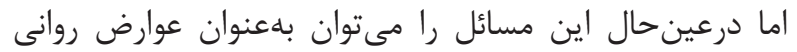

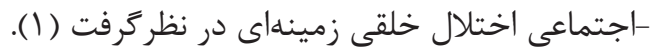

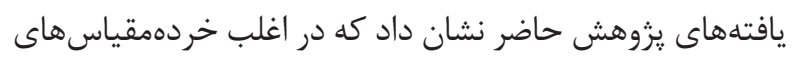

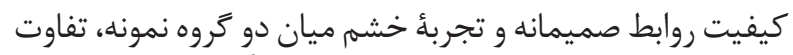

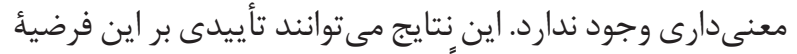

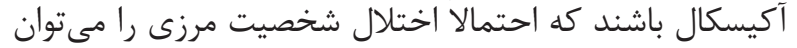

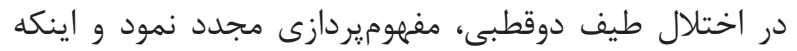

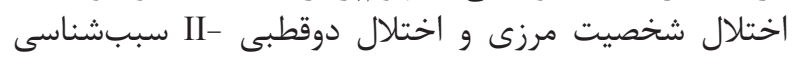

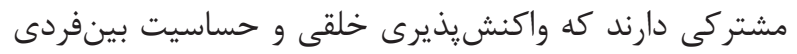

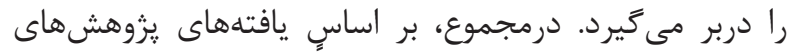

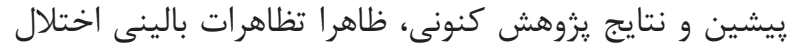

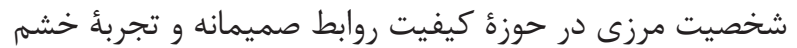

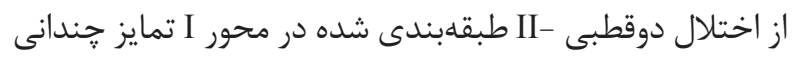

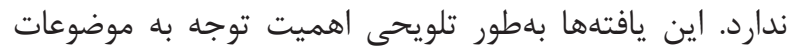

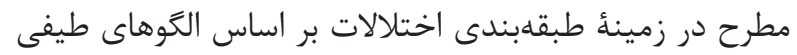

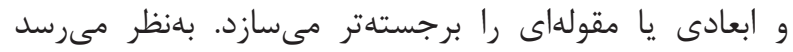

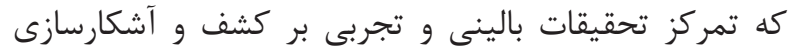

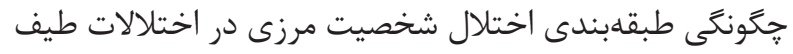

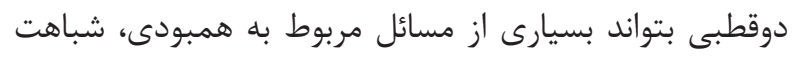

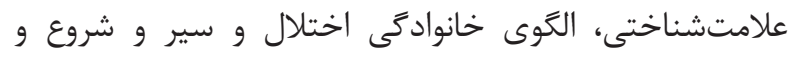

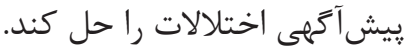

از بسيارى جهات اين يزوهش در نوع خود اطلاعات جديدى

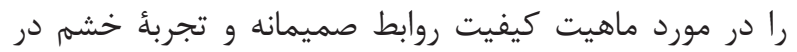

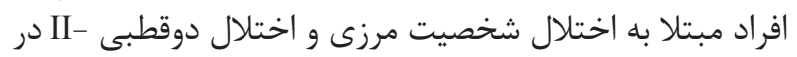

\section{${ }^{32}$ Perugi}

${ }^{33}$ Levitt

${ }^{34}$ Deltito
بحث و نتيجهة

هدف از يزوهش حاضر مقايسٔ كيفيت روابط صميمانه و تجربأ

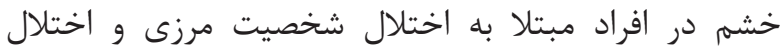

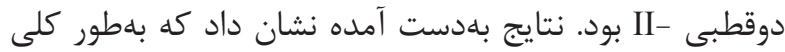

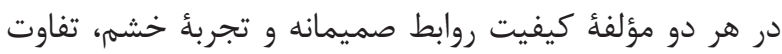

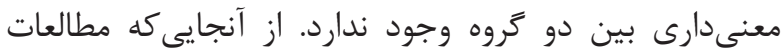

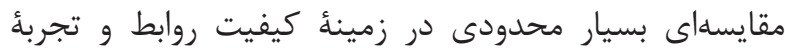

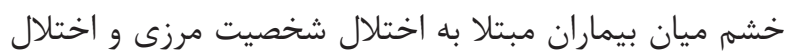

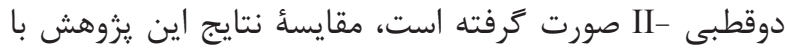

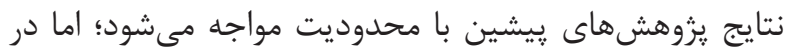

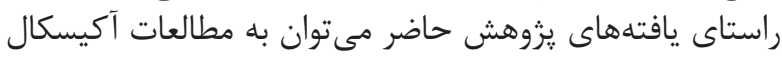

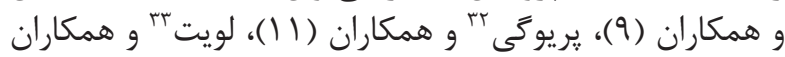

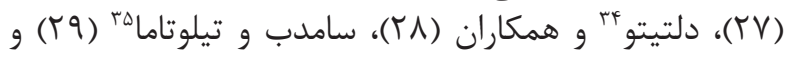

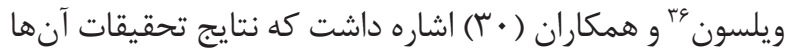

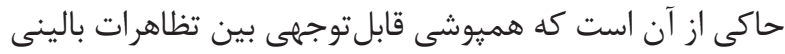

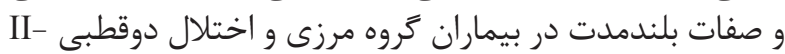

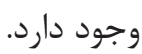

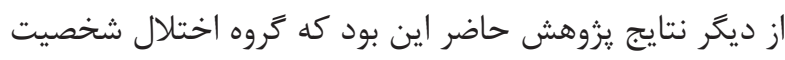

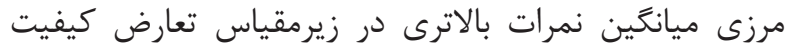

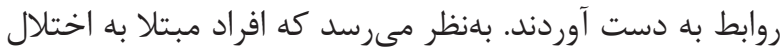

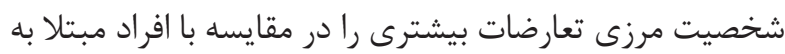

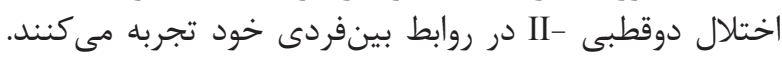

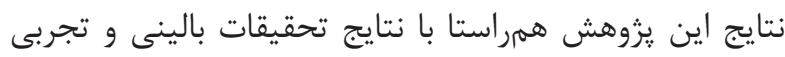

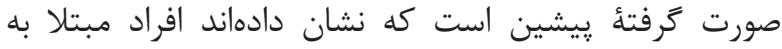

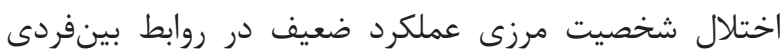

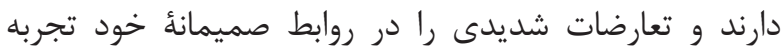

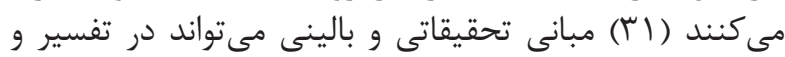
تعبير اين يافته نقش مهمى داشته تحفيقاني باشند.

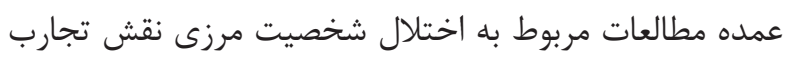

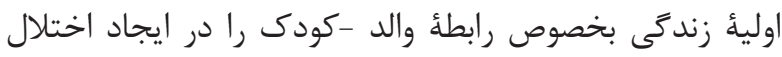

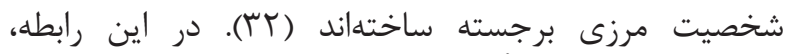

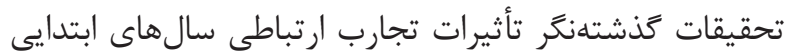

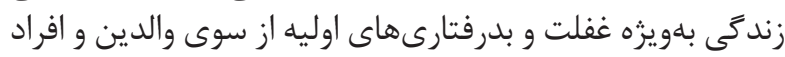

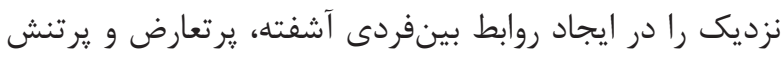

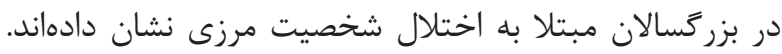

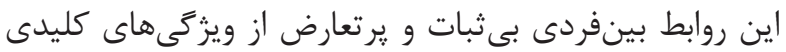

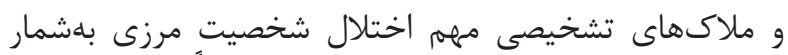

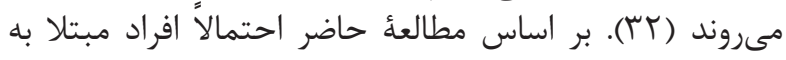

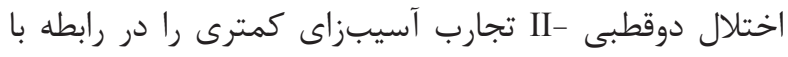
مراقبان خود از جمله والدين داشتهاند.

همسو با نتايج يزوهش حاضر كه افراد مبتلا به اختلال شخصيت

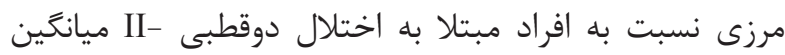

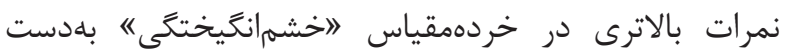

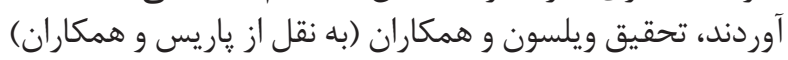

\footnotetext{
${ }^{35}$ Somdeb and Tilottama

${ }^{36}$ Wilson

${ }^{37}$ Howland and Thase
} 


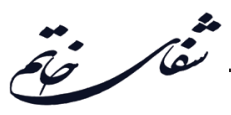

كستردهتر براى كشف تفاوت و شباهتها و ماهيت كلى روابط

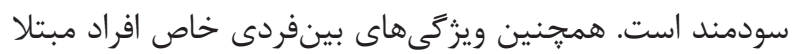

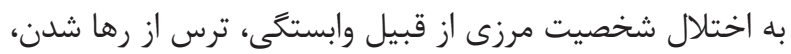

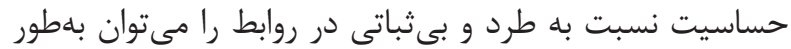
اختصاصىتر با گروه اختلال دوقطبى -III مقايسه نمود.

در حوزه هيجان و خلق نيز بررسىهاى بيشتر در زمينأ

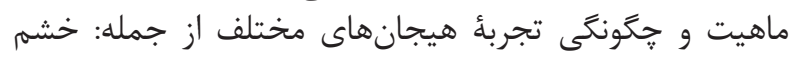

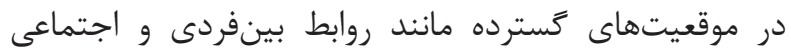

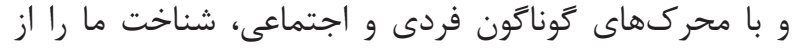

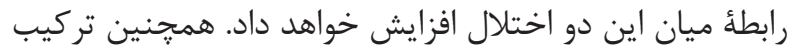

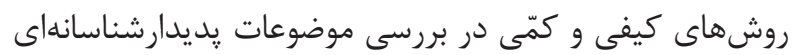

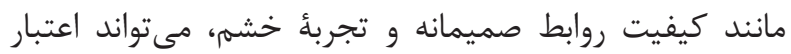

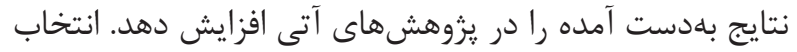

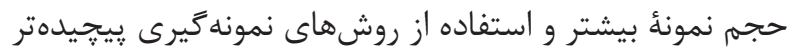

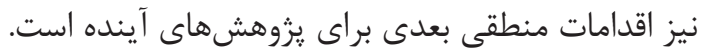

1. Howland R, Thase MA. Comprehensive review of cyclothymic disorder. J Nerv Ment Dis. 1993; 181(8): 485-93.

2. Magill A. The boundary between borderline personality disorder and bipolar disorder: current concepts and challenges. Can J Psychiatry. 2004; 49(8): 551-6.

3. Blacker D, Tsuang M. Contested boundaries of bipolar disorder and the limits of categorical diagnosis in psychiatry. Am J Psychiatry. 1998; 155(12): 1733-9.

4. Tyrer S, Brittlebank A. Misdiagnosis of bipolar affective disorder as personality disorder. Can J Psychiatry. 1993; 38(9): 587-9.

5. Zanarini MC, Frankenburg FR, Dubo ED, Sickel AE, Trikha A, Levin A. Axis I comorbidity of borderline personality disorder. Am J Psychiatry. 1998; 155(12): 1733-9.

6. Zanarini MC, Frankenburg FR, Hennen J, Reich DB, Silk KR. Axis I comorbidity in patients with borderline personality disorder: 6-year follow-up and prediction of time to remission. Am J Psychiatry. 2004; 161(11): 2108-14.

7. Gunderson J, Phillips KA. A current view of the interface between borderline personality disorder and depression. Am J Psychiatry. 1991; 148(8): 967-75.
مقايسه با يكديگر نشان داده است. با اين وجود يزوهش حاضر

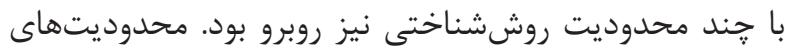

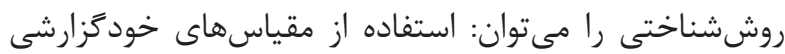

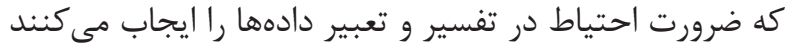
و استفاده از روش نمونه روشهاى نمونه

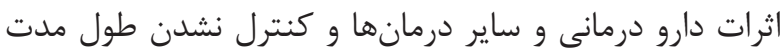

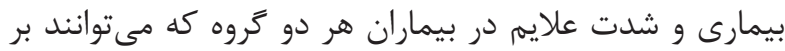

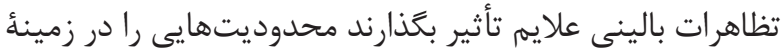

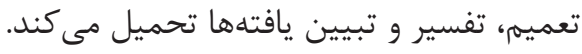

اين :زروهش، كيفيت روابط صميمانه را كه يكى از مؤلفههاى عمده

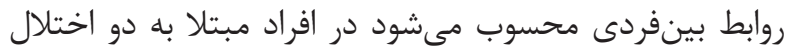

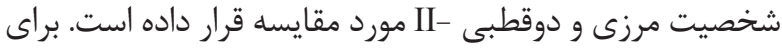

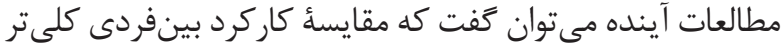

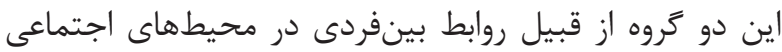

8. Koenigsberg HW, Anwunah I, New AS, Mitropoulou V, Schopick F, Siever LJ. Relationship between depression and borderline personality disorder. Depress Anxiety. 1999; 10(4): 158-67.

9. Akiskal HS, Chen SE, Davis GC, Puzantian VR, Kashgarian M, Bolinger JM. Borderline: an adjective in search of a noun. J Clin Psychiatry. 1985; 46(2): 41-8.

10. Akiskal HS, Djenderedjian AM, Rosenthal RH, Khani MK. Cyclothymic disorder: validating criteria for inclusion in the bipolar affective group. Am J Psychiatry. 1977; 134(11): 1227-33.

11. Perugi G, Toni C, Maria, Traviersoa Ch, Akiskal HS. The role of cyclothymia in atypical depression: toward a data-based reconceptualization of the borderline-bipolar II connection. J Affect Disord. 2003; 73(1-2): 87-98.

12. Akiskal HS. The bipolar spectrum-the shaping of a new paradigm in psychiatry. Curr Psychiatry Rep. 2002; 4(1): $1-3$.

13. Gunderson JG, Links PS. Borderline personality disorder: a clinical guide. 2nd ed. American Psychiatric Publishing. Inc. 2008; p. 18-20.

14. Alden SA. Borderline personality disorder and aggression: Exploring mediating and moderating roles of psychophysiological arousal. Emory University. 2008. 
15. Bauer MS, Kilbourne AM, Greenwald DE, Ludmane EJ, Mcbride L. Overcoming bipolar disorder: a comprehensive workbook for managing your symptoms and achieving your life goals. New Harbinger Publications. 2009.

16. Carolan LA, Power MJ. What basic emotions are experienced in bipolar disorder? Clin psycho psychot. 2011; 18(5): 366-78.

17. Linehan M. Cognitive-behavioral treatment of borderline personality disorder. New York: Guilford Press. 1993; p. 62-3.

18. Wright J, Turkington D, Kingdon DG, Ramirez BM. Cognitive-Behavior therapy for severe mental illness: an illustrated Guide. American psychiatric publishing inc. 2009; p. 211-5.

19. Lieberman DZ, Massey SH, Goodwin FK. The role of gender in single vs married individuals with bipolar disorder. Comp Psychiat. 2010; 51(4): 380-5.

20. Goodwin FK, Jamison K. Manic-depressive illness: bipolar disorder and recurrent depression. 2nd ed. New York: Oxford University Press. 2007.

21. Eftekhar M, Shabani A. Borderline personality disorder is not a subtype of bipolar disorder. J Psychi Cli Psycho. 2009; 15(2): 147-58.

22. Paris J, Gunderson J, Weinberg I. The interface between borderline personality disorder and bipolar spectrum disorders. Comp Psychiat. 2007; 48(2): 14554.

23. Mohammadzadeh A, Rezaei A. Validation of the borderline personality inventory in Iran. J Behav Sci. 2011; 5(3): 269-77.

24. Hormozi M. The reliability and validity of the Persian version Mood Disorder Questionnaire. PhD thesis. Tehran. Iran University of Medical Sciences,
Psychiatric Institute. 2008.

25. Hosseini Ghadamgahi J. Quality of social relationships and coping with stress in coronary heart disease. PhD thesis. Tehran. Iran University of Medical Sciences, Psychiatric Institute. 2008.

26. Besharat M, Habibnejad M. Psychometric characteristics of the multidimensional anger inventory. cont psycho. 2008; 3(2): 14-22.

27. Levitt AJ, Joffe RT, Ennis J, MacDonald C, Kutcher SP. The prevalence of cyclothymia in borderline personality disorder. J Clin Psychiat. 1990; 51(8): 335-9.

28. Deltito J, Martin L, Riefkohl J. Do patients with borderline personality disorder belong to the bipolar spectrum? J Affect Disorders. 2001; 67(1-3): 221-8.

29. Somdeb M, Tilottama M. Personality characteristics and development of psychopathology in bipolar I and borderline personality disorder patients. Psychol Stud. 2013; 58(2): 179-87.

30. Kahr NA, Jorgensen CR, Straarup KN, Licht RW. Severity of affective temperament and maladaptive self-schemas differentiate borderline patients, bipolar patients and controls. Comp Psychiat. 2010; 51(5): 48691.

31. Drapeau M, Perry CJ, Körner A. Interpersonal behaviours and BPD. Are specific interpersonal behaviours related to borderline personality disorder? An empirical study using the Core Conflictual Relationship Theme standard categories. Arch Psych. 2010; 3(3): 5-10.

32. Fonagy P. Attachment, mentalization and borderline personality disorder. Eur J Psychother couns. 2008; 8(1): 35-47.

33. La' Talova K. Bipolar disorder and aggression. Int J Clin Pract. 2009; 63(6): 889-99. 